\title{
Protective effects of hydrogen sulfide anions against acetaminophen-induced hepatotoxicity in mice
}

\author{
Isao Ishii ${ }^{1}$, Shotaro Kamata', Yoshifumi Hagiya' ${ }^{1}$, Yumi Abiko², Tadashi Kasahara ${ }^{1}$ \\ and Yoshito Kumagai² \\ ${ }^{1}$ Department of Biochemistry, Keio University Graduate School of Pharmaceutical Sciences, \\ 1-5-30 Shibakoen, Minato-ku, Tokyo 105-8512, Japan \\ ${ }^{2}$ Graduate School of Comprehensive Human Sciences, University of Tsukuba, \\ 1-1-1 Tennodai, Tsukuba, Ibaraki 305-8575, Japan
}

(Received August 26, 2015; Accepted September 28, 2015)

\begin{abstract}
The key mechanism for hepatotoxicity resulting from acetaminophen (APAP) overdose is cytochrome P450-dependent formation of $N$-acetyl- $p$-benzoquinone imine (NAPQI), a potent electrophilic metabolite that forms protein adducts. The fundamental roles of glutathione in the effective conjugation/clearance of NAPQI have been established, giving a molecular basis for the clinical use of $\mathrm{N}$-acetylcysteine as a sole antidote. Recent evidence from in vitro experiments suggested that sulfide anions ( $\mathrm{S}^{-}$) to yield hydrogen sulfide anions (HS-) under physiological $\mathrm{pH}$ could effectively react with NAPQI. This study evaluated the protective roles of $\mathrm{HS}^{-}$against APAP-induced hepatotoxicity in mice. We utilized cystathionine $\gamma$-lyase-deficient $\left(\mathrm{Cth}^{--}\right)$mice that are highly sensitive to acetaminophen toxicity. Intraperitoneal injection of acetaminophen $(150 \mathrm{mg} / \mathrm{kg})$ into $\mathrm{Cth}^{-/}$mice resulted in highly elevated levels of serum alanine/aspartate aminotransferases and lactate dehydrogenase associated with marked increases in oncotic hepatocytes; all of which were significantly inhibited by intraperitoneal preadministration of sodium hydrosulfide (NaHS). NaHS preadministration significantly suppressed APAP-induced serum malondialdehyde level increases without abrogating APAP-induced rapid depletion of hepatic glutathione. These results suggest that exogenous HS- protects hepatocytes by directly scavenging reactive NAPQI rather than by increasing cystine uptake and thereby elevating intracellular glutathione levels, which provides a novel therapeutic approach against acute APAP poisoning.
\end{abstract}

Key words: Acetaminophen, $N$-acetyl- $p$-benzoquinone imine, Glutathione, Hydrogen sulfide, Hepatotoxicity

\section{INTRODUCTION}

Acetaminophen (APAP: $N$-acetyl- $p$-aminophenol; also known as paracetamol) is the most popular analgesic/ antipyretic drug. It is generally safe and free of adverse effects at recommended doses. However, APAP overdose is a frequent cause of liver failure and the leading cause of death due to deliberate or accidental overdose in developed countries (Bromer and Black, 2003; Lee, 2008). The majority of orally administrated APAP is glucuronidated or sulfated by the phase II metabolic pathway in the liver, leading to urinary excretion, whereas a small proportion $(\sim 10 \%)$ is metabolized by certain cytochrome P450 isoforms to $N$-acetyl- $p$-benzoquinone imine (NAPQI), a highly reactive electrophile that forms covalent pro- tein adducts and thereby causes hepatocellular necrosis (Hinson et al., 2010).

Fundamental roles of intracellular glutathione (GSH) in protection against APAP/NAPQI toxicity have been well established; glutathione $S$-transferase is capable of rapidly detoxifying NAPQI by catalyzing conjugation with reduced GSH. Indeed, NAPQI binding to proteins was not observed in hepatocytes until the complete depletion of GSH (Mitchell et al., 1973). In accordance with this, we demonstrated that mice lacking cystathionine $\gamma$-lyase (CTH), the final transsulfuration enzyme that is essential for cysteine biosynthesis and thus efficient GSH biosynthesis, were highly susceptible to APAP-induced hepatic injury because of their rapid hepatic GSH depletion upon APAP administration (Hagiya et al., 2015). Mean-

Correspondence: Isao Ishii (E-mail: isao-ishii@umin.ac.jp) 
while, CTH is also known as a hydrogen sulfide $\left(\mathrm{H}_{2} \mathrm{~S}\right) /$ reactive persulfides (polysulfides)-producing enzyme (Kimura, 2015; Ono et al., 2014; Ida et al., 2014). $\mathrm{H}_{2} \mathrm{~S}$ could exert its cytoprotective effects by increasing cellular cyst(e)ine uptake and activating glutathione synthesis (Kimura et al., 2010) whereas aqueous sulfide anions $\left(\mathrm{S}^{2-}\right)$ or polysulfides (sodium polysulfides as well as GSH polysulfides) could trap NAPQI by forming sulfur adducts (Trettin et al., 2014; Abiko et al., 2015). Therefore, the increased susceptibility of $\mathrm{Cth}^{-/-}$mice to APAP hepatotoxicity could be attributable, at least in part, to their decreased production of such endogenous $\mathrm{H}_{2} \mathrm{~S} /$ reactive persulfide (polysulfide) species. In this study, we examined whether the simplest $\mathrm{H}_{2} \mathrm{~S}$ anion donor (NaHS) could confer cytoprotective effects against APAP-induced severe hepatic injury in mice independent of their hepatic GSH level increase.

\section{MATERIALS AND METHODS}

\section{Animals}

CTH hemizygous knockout $\left(\mathrm{Cth}^{+/-}\right)$male or female mice on a C57BL/6J inbred strain (CLEA Japan, Tokyo, Japan) were bred to obtain $\mathrm{Cth}^{-/-}$female mice (Ishii et al., 2010). Mice were housed in an air-conditioned room kept on a 12-hr dark/light ( 8 pm-8 am) cycle, and allowed free access to a standard dry rodent diet and water. All animal procedures conformed to the Guide for the Care and Use of Laboratory Animals, Eighth Edition published by the US National Research Council, and were approved by the Animal Care Committee of Keio University (No. 09187(4)).

\section{APAP and NaHS injection}

Adult (8-10-week-old) female mice were fasted for $20 \mathrm{hr}$ (3 pm-11 am) and then injected intraperitoneally with APAP (150 mg/kg; Sigma A7302) dissolved in saline (Hagiya et al., 2015). After $4 \mathrm{hr}$, mice were anesthetized with isoflurane and blood/liver samples were collected. In some experiments, mice were preadministrated intraperitoneally with NaHS (18.7 mg/kg; Sigma 161257) dissolved in saline $2 \mathrm{hr}$ prior to APAP injection.

\section{Serum biochemical analysis}

Serum levels of alanine aminotransferase (ALT), aspartate aminotransferase (AST), and lactate dehydrogenase (LDH) were measured using a Dri-Chem 7000i biochemistry analyzer (Fujifilm, Tokyo, Japan). Serum levels of malondialdehyde (MDA) were measured using a TBARS (thiobarbituric acid-reactive substances) assay kit (Cayman Chemical, Ann Arbor, MI, USA) (Yamada et al., 2012).

\section{Histochemistry}

Livers were quickly dissected out, fixed in $10 \%$ formalin, dehydrated in an ascending ethanol series (from $75 \%$ to $100 \%$ ) and then xylene, and embedded in paraffin. Three-micrometer sections were cut, deparaffinized, and stained using an Apop Tag Peroxidase In Situ Apoptosis Detection kit (EMD Millipore, Billerica, MA, USA) for terminal deoxynucleotidyl transferase dUTP nick end labeling (TUNEL) (Hagiya et al., 2015). The slides were examined using a BZ-9000 microscope (Keyence, Osaka, Japan) fitted with Plan Apo $4 \times$ and $10 \times$ objective lenses (Nikon, Tokyo, Japan).

\section{Hepatic total glutathione measurement}

Liver aliquots were homogenized in 5\% 5-sulfosalicylic acid and the homogenates were centrifuged at $4{ }^{\circ} \mathrm{C}$ for $10 \mathrm{~min}$ at $10,000 \mathrm{~g}$. Then the supernatants were centrifuged at $4^{\circ} \mathrm{C}$ for $10 \mathrm{~min}$ at $10,000 \mathrm{~g}$. Levels of total GSH (GSH + oxidized glutathione [GSSG]) in the final supernatant were measured using a GSSG/GSH Quantification kit (Dojindo, Kumamoto, Japan) (Hagiya et al., 2015).

\section{Statistical analysis}

Data are means \pm S.D. ( $n$ as indicated). Statistical analyses were performed using two-tailed unpaired Student's $t$-test, where $P$ values of 0.05 or less were considered significant.

\section{RESULTS AND DISCUSSION}

APAP injection $(150 \mathrm{mg} / \mathrm{kg})$ dramatically elevated serum levels of ALT $(\sim 30,000 \mathrm{IU} / \mathrm{L}), \mathrm{AST}$ ( 40,000 IU/L), and LDH ( 100,000 IU/L) in $\mathrm{Cth}^{-/-}$mice but much less $(30,159,335 \mathrm{IU} / \mathrm{L}$, respectively) in wildtype mice within $4 \mathrm{hr}$ (Fig. 1A-C), as we reported previously with high serum ALT levels in APAP-injected $\mathrm{Cth}^{-/}$ (not wild-type) mice (Hagiya et al., 2015). Administration of NaHS (18.7 mg/kg; $500 \mu \mathrm{M}$ estimate in body fluid) alone did not affect serum ALT/AST/LDH levels in wildtype or $\mathrm{Cth}^{-/-}$mice; however, it suppressed APAP-induced ALT/AST/LDH elevation in $C t h^{-/-}$mice (Fig. 1A-C) although not significantly as for LDH (Fig. 1C). Administration of NaHS slightly elevated levels of serum ALT (but not those of AST/LDH significantly) in APAP-injected WT mice (Fig. 1A-C); the reason for this is unknown. APAP overdose is known to cause oncotic necrosis (oncosis) that is distinguishable from caspase 3-mediated apoptosis but is detectable by TUNEL staining; TUNEL staining also detects oncotic nuclei that are randomly fragment- 
HS- $^{-}$anions ameliorate acetaminophen hepatotoxicity in mice

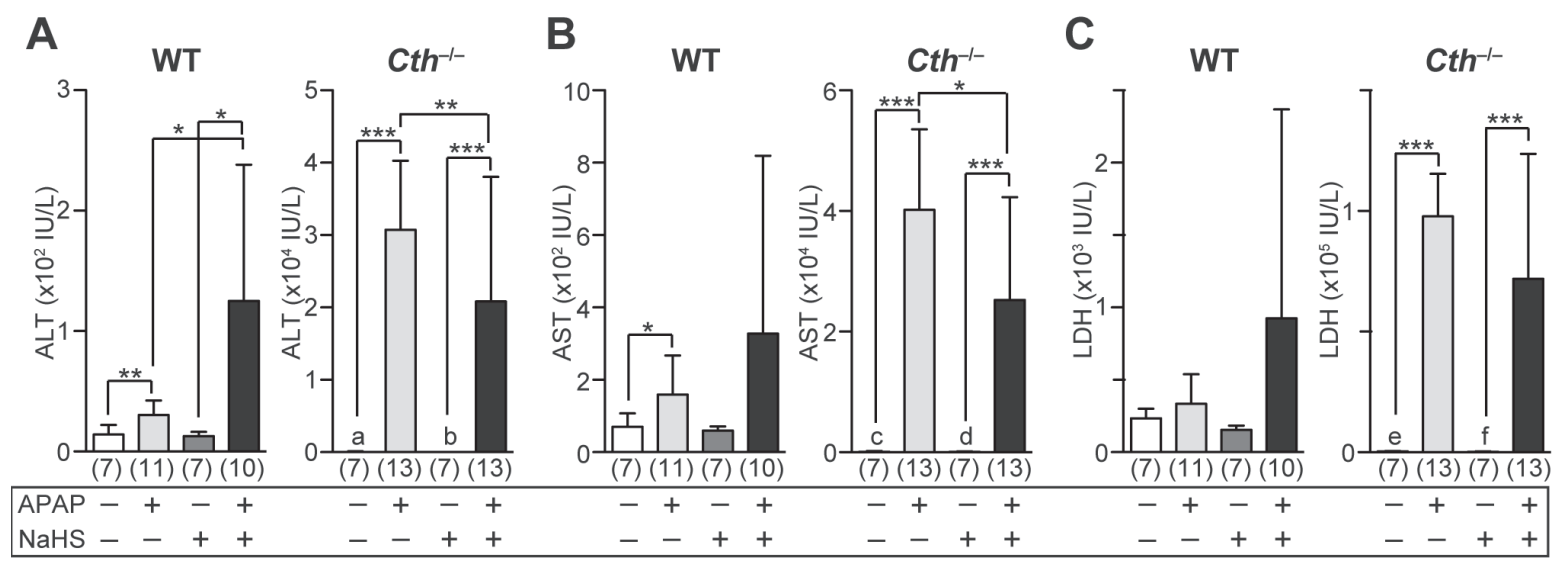

Fig. 1. Protective effects of sodium hydrosulfide (NaHS) on acetaminophen (APAP)-induced hepatic injury. Wild-type (WT) and $C t h^{-1-}$ mice were fasted for $18 \mathrm{hr}$ and then injected with NaHS $(18.7 \mathrm{mg} / \mathrm{kg})$ or saline. Two hours later, the mice were injected with APAP $(150 \mathrm{mg} / \mathrm{kg})$ or saline. After $4 \mathrm{hr}$, the mice were anesthetized and blood samples were collected. Serum levels of ALT (A), AST (B), and LDH (C) were measured. Data are means \pm S.D. ( $n$ : sample number); ${ }^{*} P<0.05,{ }^{* *} P<0.01$, and ${ }^{* * *} P<0.001$ by $t$-test. Some data (bars and error bars) are not seen for APAP-non-injected $\mathrm{Cth}^{-/-}$mouse samples (a-f) because of their much lower levels compared to those for APAP-injected $C t h^{-/-}$mouse samples; actual values are $13.2 \pm 5.5$ for a, $15.4 \pm 6.1$ for b, $114 \pm 95$ for c, $98 \pm 52$ for d, $360 \pm 209$ for e, and $281 \pm 127$ for $\mathrm{f}$ (all in IU/L).

ed by $\mathrm{Ca}^{2+}$-dependent/caspase-independent endonucleases (Hagiya et al., 2015; Jaeschke et al., 2012). TUNEL staining of hepatic sections identified chromatin-clumped nuclei as well as the cytoplasm of oncotic hepatocytes around the centrilobular regions (both brown-colored) (Hagiya et al. 2015) in both APAP- and APAP/ NaHS-injected $\mathrm{Cth}^{-/-}$mice, but the TUNEL-positive areas were much smaller when mice were preadministrated with NaHS (Fig. 2A-C). In representative hepatic sections, TUNEL-positive areas were $56.6 \pm 6.5 \%(n=3)$ of whole section areas in APAP/NaHS-injected $\mathrm{Cth}^{-/-}$mice, whereas $67.5 \pm 3.9 \%(n=3)$ in APAP-injected $C t h^{-/}$mice $(P=0.082$ by $t$-test). In contrast, TUNEL-positive cells were not found in hepatic sections from APAP alone-injected (Fig. 2D) or APAP/NaHS-injected (Fig. 2E) wildtype mice.

Several working hypotheses have been proposed for the cytoprotective actions of $\mathrm{H}_{2} \mathrm{~S}$ /polysulfides (Kimura, 2015). $\mathrm{H}_{2} \mathrm{~S}$ may somehow potentiate the activity of the cystine/glutamate antiporter as well as the cysteine transporter and enhance the activity of glutamate-cysteine ligase, a rate-limiting enzyme of GSH synthesis; both of which could contribute for increased intracellular GSH levels to protect cells from oxidative toxicity (Kimura et al., 2010). On the other hand, polysulfides (but not $\mathrm{H}_{2} \mathrm{~S}$ itself probably) may sulfhydrate particular cysteine residues in various functional proteins, thereby regulat- ing their functions (Kimura, 2015; Ono et al., 2014; Ida et al., 2014). We demonstrated previously that APAP induced rapid depletion of hepatic GSH levels and elevation of serum MDA levels in $\mathrm{Cth}^{-/-}$mice but not in wild-type mice (Hagiya et al., 2015). In this study, NaHS preadministration failed to abolish APAP-induced rapid depletion of hepatic GSH levels (Fig. 3A) but significantly reduced APAP-induced serum MDA levels (Fig. 3B). These results suggested that exogenous HS ${ }^{-}$anions directly scavenged reactive NAPQI without increasing cystine uptake and intracellular GSH levels. This is supported by Trettin et al. who detected in vitro formation of NAPQI sulfur adducts in dog liver lysates mixed with APAP/ $\mathrm{Na}_{2} \mathrm{~S}$ as well as liver lysates of APAP-injected mice mixed with $\mathrm{Na}_{2} \mathrm{~S}$ (Trettin et al., 2014), or by our group that detected in vivo formation of NAPQI sulfur adducts in the liver/serum/urine of APAP-injected mice as well as in vitro formation of $\mathrm{NAPQIH}_{2}-\mathrm{SH}, \mathrm{NAPQIH}_{2}-\mathrm{S}-$ $\mathrm{NAPQIH}_{2}$ and $\mathrm{NAPQIH}_{2}-\mathrm{SS}-\mathrm{NAPQIH}{ }_{2}$ during the reaction between NAPQI and $\mathrm{Na}_{2}{ }^{32} \mathrm{~S}$ (or $\mathrm{Na}_{2}{ }^{34} \mathrm{~S}$ ) by LC-MS/ MS analysis (Abiko et al., 2015). All these results suggested that NAPQI sulfur adducts would be produced in mice given APAP and NaHS.

A previous study mentioned the curative effects of NaHS (3 mg/kg) against APAP (650 mg/kg)-induced mild hepatotoxicity (ALT: from $\sim 325$ to $\sim 70$ in IU/L) in outbred Swiss male mice in which NaHS administra- 

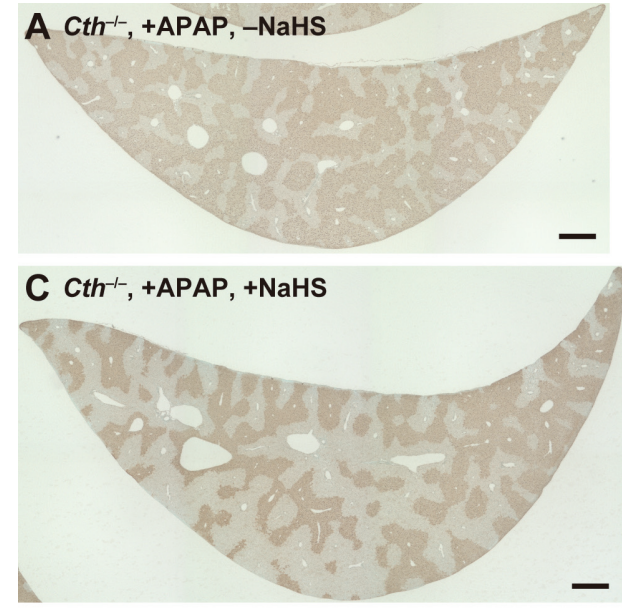

D WT, +APAP, -NaHS
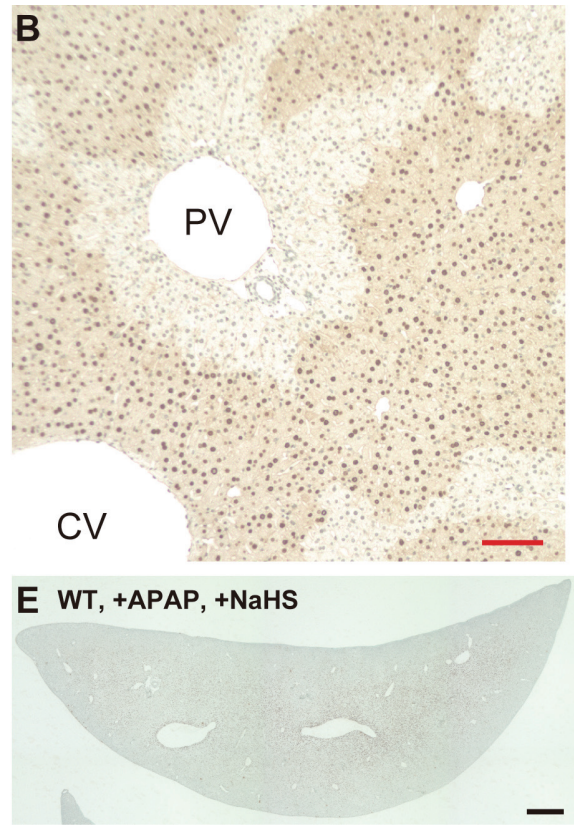

Fig. 2. Protective effects of sodium hydrosulfide (NaHS) on acetaminophen (APAP)-induced hepatic injury. Cth-/ (A-C) and wildtype (WT; D and E) mice were fasted for $18 \mathrm{hr}$ and then injected with saline (A and D) or NaHS (18.7 mg/kg; C and E). Two hours later, all mice were injected with APAP $(150 \mathrm{mg} / \mathrm{kg})$. Paraffin-embedded hepatic sections were stained for terminal deoxynucleotidyl transferase dUTP nick end labeling (TUNEL). B is the microscopic magnification of a part of A. Bars: $500 \mu \mathrm{m}$ (black) or $100 \mu \mathrm{m}($ red $)$. CV, central vein; PV, portal vein.

A

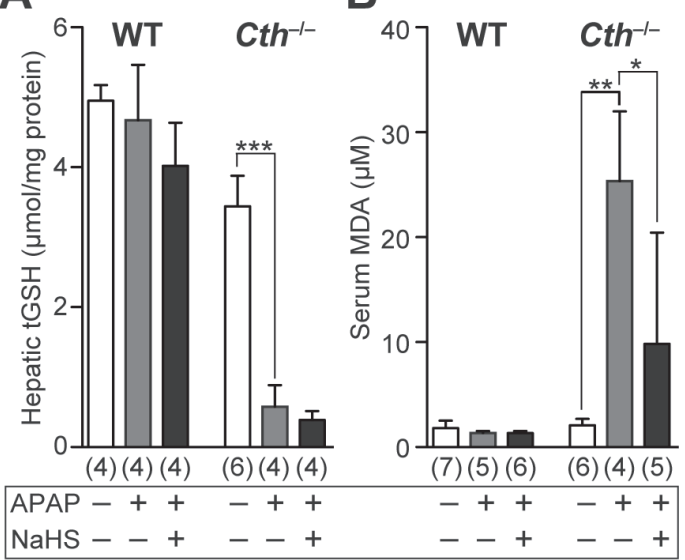

Fig. 3. Effects of sodium hydrosulfide (NaHS) on acetaminophen (APAP)-induced hepatic glutathione (GSH) depletion and serum malondialdehyde (MDA) level increase. Wild-type (WT) and $\mathrm{Cth}^{-/}$mice were fasted for $18 \mathrm{hr}$ and then injected with NaHS $(18.7 \mathrm{mg} /$ $\mathrm{kg}$ ) or saline. Two hours later, the mice were injected with APAP $(150 \mathrm{mg} / \mathrm{kg})$ or saline. After $4 \mathrm{hr}$, the mice were anesthetized and liver/blood samples were collected. Hepatic GSH levels (A) and serum MDA levels (B) were measured. Data are means \pm S.D. ( $n$ as indicated), and effects of APAP or NaHS are significant at ${ }^{*} P<0.05,{ }^{* *} P<0.01$, and ${ }^{* * *} P<0.001$ by $t$-test. tion significantly increased hepatic GSH levels (Morsy et al., 2010). Therefore, the sites of HS- action may alter depending on the levels of APAP toxicity. Obviously, our experiments are more related to the clinical setting of (occasionally lethal) severe acute liver failure due to APAP overdose. Although $N$-acetylcysteine has been used for the treatment with APAP overdose, early treatment is recommended because it requires the hepatic activities of glutamate-cysteine ligase and glutathione synthase for its conversion to GSH. Our study suggests that $\mathrm{H}_{2} \mathrm{~S}$ / polysulfide treatment could be the final remedy for terminal patients with APAP overdose by capturing NAPQI formed from APAP, resulting in its sulfur adducts such as $\mathrm{NAPQIH}_{2}$-cysteine trisulfide adduct and $\mathrm{NAPQIH}_{2}-\mathrm{GSH}$ persulfide adduct (Abiko et al., 2015). We unfortunately failed to obtain effective hepatoprotection using NaHS postadministration after APAP injection in our $\mathrm{Cth}^{-1-}$ mice (data not shown), but $\mathrm{H}_{2} \mathrm{~S}$ /polysulfide postadministration with $N$-acetylcysteine could be beneficial. Further trials using newly developed several polysulfide donors are also awaited. 
HS $^{-}$anions ameliorate acetaminophen hepatotoxicity in mice

\section{ACKNOWLEDGMENTS}

This work was supported in part by the Grants-in-Aid for Scientific Research (25460072 to I.I. and 25220103 to Y.K.) and the Program for Strategic Research Foundation at Private Universities (S1101003 to I.I.) from the Ministry of Education, Culture, Sports, Science and Technology of Japan; Program for Advancement of Next Generation Research Projects and Fukuzawa Memorial Fund for the Advancement of Education and Research from Keio University (to I.I.).

Conflict of interest---- The authors declare that there is no conflict of interest.

\section{REFERENCES}

Abiko, Y., Ishii, I., Kamata, S., Tsuchiya, Y., Watanabe, Y., Ihara, H., Akaike, T. and Kumagai, Y. (2015): Formation of sulfur adducts of $\mathrm{N}$-acetyl-p-benzoquinoneimine, an electrophilic metabolite of acetaminophen in vivo: Participation of reactive persulfides. Chem. Res. Toxicol., 28, 1796-1802.

Bromer, M.Q. and Black, M. (2003): Acetaminophen hepatotoxicity. Clin. Liver Dis., 7, 351-367.

Hagiya, Y., Kamata, S., Mitsuoka, S., Okada, N., Yoshida, S., Yamamoto, J., Ohkubo, R., Abiko, Y., Yamada, H., Akahoshi, N., Kasahara, T., Kumagai, Y. and Ishii, I. (2015): Hemizygosity of transsulfuration genes confers increased vulnerability against acetaminophen-induced hepatotoxicity in mice. Toxicol. Appl. Pharmacol., 282, 195-206.

Hinson, J.A., Roberts, D.W. and James, L.P. (2010): Mechanisms of acetaminophen-induced liver necrosis. Handb. Exp. Pharmacol., 196, 369-405.

Ida, T., Sawa, T., Ihara, H., Tsuchiya, Y., Watanabe, Y., Kumagai, Y., Suematsu, M., Motohashi, H., Fujii, S., Matsunaga, T., Yamamoto, M., Ono, K., Devarie-Baez, N.O., Xian, M., Fukuto, J.M. and Akaike, T. (2014): Reactive cysteine persulfides and S-polythi- olation regulate oxidative stress and redox signaling. Proc. Natl. Acad. Sci. USA, 111, 7606-7611.

Ishii, I., Akahoshi, N., Yamada, H., Nakano, S., Izumi, T. and Suematsu, M. (2010): Cystathionine gamma-lyase-deficient mice require dietary cysteine to protect against acute lethal myopathy and oxidative injury. J. Biol. Chem., 285, 26358-26368.

Jaeschke, H., McGill, M.R. and Ramachandran, A. (2012): Oxidant stress, mitochondria, and cell death mechanisms in drug-induced liver injury: lessons learned from acetaminophen hepatotoxicity. Drug Metab. Rev., 44, 88-106.

Kimura, H. (2015): Hydrogen sulfide and polysulfides as signaling molecules. Proc. Jpn. Acad. Ser. B Phys. Biol. Sci., 91, 131-159.

Kimura, Y., Goto, Y.I. and Kimura, H. (2010): Hydrogen sulfide increases glutathione production and suppresses oxidative stress in mitochondria. Antioxid. Redox Signal., 12, 1-13.

Lee, W.M. (2008): Acetaminophen-related acute liver failure in the United States. Hepatol. Res., 38 Suppl 1, S3-S8.

Mitchell, J.R., Jollow, D.J., Potter, W.Z., Gillette, J.R. and Brodie, B.B. (1973): Acetaminophen-induced hepatic necrosis. IV. Protective role of glutathione. J. Pharmacol. Exp. Ther., 187, 211217.

Morsy, M.A., Ibrahim, S.A., Abdelwahab, S.A., Zedan, M.Z. and Elbitar, H.I. (2010): Curative effects of hydrogen sulfide against acetaminophen-induced hepatotoxicity in mice. Life Sci., 87, 692-698.

Ono, K., Akaike, T., Sawa, T., Kumagai, Y., Wink, D.A., Tantillo, D.J., Hobbs, A.J., Nagy, P., Xian, M., Lin, J. and Fukuto, J.M. (2014): Redox chemistry and chemical biology of H2S, hydropersulfides, and derived species: Implications of their possible biological activity and utility. Free Radic. Biol. Med., 77, 82-94.

Trettin, A., Batkai, S., Thum, T., Jordan, J. and Tsikas, D. (2014): Trapping of NAPQI, the intermediate toxic paracetamol metabolite, by aqueous sulfide $\left(\mathrm{S}^{2-}\right)$ and analysis by GC-MS/MS. J. Chromatogr. B Analyt. Technol. Biomed. Life Sci., 963, 99-105.

Yamada, H., Akahoshi, N., Kamata, S., Hagiya, Y., Hishiki, T., Nagahata, Y., Matsuura, T., Takano, N., Mori, M., Ishizaki, Y., Izumi, T., Kumagai, Y., Kasahara, T., Suematsu, M. and Ishii, I. (2012): Methionine excess in diet induces acute lethal hepatitis in mice lacking cystathionine $\gamma$-lyase, an animal model of cystathioninuria. Free Radic. Biol. Med., 52, 1716-1726. 